\title{
Mechanisms of CXCR7 induction in malignant melanoma development
}

\author{
XIAO-JING LI ${ }^{1}$, PAI LIU ${ }^{1,2}$, WEI-WEI TIAN ${ }^{2}$, ZHI-FENG LI ${ }^{1}$, BAO-GUO LIU ${ }^{1}$ and JIAN-FANG SUN ${ }^{3}$ \\ ${ }^{1}$ Department of Dermatology, Affiliated Hospital of Hebei University of Engineering, Handan, Hebei 056002; \\ ${ }^{2}$ Department of Dermatology, Jiangxi Province Dermatosis Special Hospital, Nanchang, Jiangxi 330000; \\ ${ }^{3}$ Institute of Dermatology, Chinese Academy of Medical Sciences, Nanjing, Jiangsu 210042, P.R. China
}

Received October 10, 2015; Accepted March 23, 2017

DOI: $10.3892 / \mathrm{ol} .2017 .6720$

\begin{abstract}
Malignant melanoma (MM) is a highly malignant skin tumor. The mechanism of MM pathogenesis and its signaling pathways are not well characterized. C-X-C chemokine receptor type 7 (CXCR7) has been reported to regulate cancer cell invasion. The present study sought to investigate the effects of CXCR7 on MM development. First, CXCR7 expression levels were assessed in the skin tumor tissue of patients with MM. Then, CXCR7 small hairpin RNA was used in M14 melanoma cells in a Transwell culture model and in a transplanted mouse model to test the effects of CXCR7. In addition, immunohistochemistry staining, reverse transcription-quantitative polymerase chain reaction and western blotting were used. The results revealed that CXCR7 expression levels were significantly higher in MM tissue compared with squamous cell carcinoma or basal cell carcinoma tissue. Knocking down CXCR7 in M14 cells significantly inhibited cell migration and invasion in the Transwell culture model. Furthermore, CXCR7 knockdown also significantly reduced the transplanted tumor size, weight and vascular number in the mouse model. It was concluded that CXCR7 interacts with C-X-C motif chemokine ligand 12 to activate the chemokine receptor signaling pathway, and to increase melanoma cell migration, invasion and development.
\end{abstract}

\section{Introduction}

Cutaneous melanoma or malignant melanoma (MM) is a highly malignant skin tumor that develops from melanocytes (1-3). MM was considered uncommon previously, but its incidence

Correspondence to: Dr Pai Liu, Department of Dermatology, Affiliated Hospital of Hebei University of Engineering, 81 Cong Tai Road, Handan, Hebei 056002, P.R. China

E-mail: zlmdsh@126.com

Dr Jian-Fang Sun, Institute of Dermatology, Chinese Academy of Medical Sciences, 12 Jiang Wang Temple Street, Nanjing, Jiangsu 210042, P.R. China

E-mail: fangmin5758@yahoo.com.cn

Key words: malignant melanoma, small hairpin RNA, CXCR7, invasion has increased remarkably over the past 30 years (4). Surgery to remove the tumor and chemotherapy are the routine treatments for early-stage melanoma (5). However, due to the high degree of genetic instability and mutations, and less sensitivity to classical chemotherapeutic treatments, there is high rate of cancer spreading to other parts of the body in patients with MM $(1,4,6)$. Therefore, the present study explored the possible mechanism of MM pathogenesis and its signaling pathways.

Chemokines are cell cytokines induced by inflammation (7). Chemokines and their signaling receptors have been shown to regulate cellular migration, cell invasion, motility, cell growth, cellular interactions with the extracellular matrix and tumor development $(8,9)$. C-X-C chemokine receptor type 7 (CXCR7) is a protein that interacts with $\mathrm{C}-\mathrm{X}-\mathrm{C}$ motif chemokine ligand 12 (CXCL12), also known as stromal cell-derived factor 1 (SDF-1), and CXCL11 in signal transduction (10). CXCR7 expression is associated with increased pathological inflammation and tumor development (10). The CXCR7-CXCL12 complex serves an important role in tumor invasion and organ-specific metastasis (10). However, the roles of CXCR7 in skin cancer invasion, tumorigenesis and tumor development are still not well understood.

Squamous cell carcinoma (SCC), basal cell carcinoma (BCC) and MM are three common skin tumors $(3,11,12)$. The present study explored the effects of CXCR7 on melanoma progression and development in the tumor tissue of human patients with MM, cultured cell lines and transplanted tumor mouse models.

Transwell chamber assays have been used for cell migration and homing assays $(13,14)$. The porous polycarbonate membrane at the bottom of the upper chamber has certain permeability. However, inactivated cells cannot pass the membrane freely. Activated migrating cells possess the ability to pass through the polycarbonate membrane by secreting hydrolytic enzymes to dissolve the porous basement membrane and bind to the matrigel membrane in the bottom chamber (15). The present study used this Transwell system to test the CXCR7 effects on melanoma cell migration and invasion.

\section{Materials and methods}

Patients and specimen collection. Human skin tumors from all selected patients were collected from January 2008 to 
December 2014 at the Institute of Dermatology, Chinese Academy of Medical Sciences (Nanjing, China) and the Affiliated Hospital of Hebei University (Hebei, China). The Chinese Academy of Medical Sciences (Nanjing, China) and the Affiliated Hospital of Hebei University (Hebei, China) Institutional Review Boards approved the present study and the protocol. Consent for surgery was obtained from all patients upon informing them of the nature of the disease, its treatment options and its possible outcomes. Classification into squamous SCC, BCC or MM was determined by histopathology through immunohistochemistry staining. Tumor tissues from 30 cases of skin SCC were harvested. The clinical profiles of patients with SCC included 16 males and 14 females, aged 34-82 years old (median age, 61.20 years). The tumor progression time ranged from 1 month to 25 years (median, 2.78 years). Among these SCC tumor cases, 12 were located on the head and face, 1 on the back, 6 on the hands, 9 on the limbs and 2 on the genital area. Among these SCC cases, $18(60 \%)$ were located on exposed parts of the body (i.e., head, face and hands) and $12(40 \%)$ on non-exposed parts. These patients included 12 cases of high-differentiated SCC (SCC-H) and 18 cases of low-differentiated SCC (SCC-L). In addition, 25 cases of skin BCC were collected, comprising 10 males and 15 females, aged 33-80 years old (mean age, 62.12 years). The tumor progression time for BCC ranged from 2 months to 11 years (median, 32.78 months). There were $17(68 \%)$ cases on exposed parts of the body and $8(32 \%)$ on non-exposed parts. A total of 30 cases of invasive skin MM samples were collected. Among these, 18 were males and 12 females, aged 35-75 years old (median age, 61.26 years), and the tumor progression time ranged from 3 to 19 months (median, 12.10 months).

Immunohistochemistry staining. CXCR7 protein was detected by immunohistochemistry. The tumor tissues were paraffin embedded and sliced into $\sim 4-\mu \mathrm{m}$ thick sections. Sections were deparaffinized with xylene and rehydrated in decreasing concentrations of ethanol. Sections were then heated in a microwave for $20 \mathrm{~min}$ in $10 \mathrm{mM}$ sodium citrate buffer for antigen retrieval. Sections were next incubated with a CXCR7 antibody (cat no., PA3-069, Thermo Fisher Scientific, Inc., Waltham, MA, USA; dilution, 1:2,000) at $4^{\circ} \mathrm{C}$ overnight. Following three washes in PBS, a peroxidase-conjugated secondary antibody (cat. no., P447, Dako; Agilent Technologies, Inc., Santa Clara, CA, USA; dilution, 1:5,000) followed by Pierce DAB Substrate kit (cat. no., 34002, Invitrogen; Thermo Fisher Scientific, Inc.) were applied for 30 and $3 \mathrm{~min}$, respectively, at room temperature. Next, sections were dehydrated in decreasing concentrations of ethanol and cover slipped. Negative control sections were incubated with an anti-mouse immunoglobulin $\mathrm{G}$ ( $\mathrm{IgG}$ ) antibody (cat. no., sc-2025; Santa Cruz Biotechnology, Inc., Dallas, TX, USA; dilution, 1:5,000). The stained sections were examined with a light microscope (IX51; Olympus Corporation, Tokyo, Japan) and photographed. MaxVision software (version 3.0; MaxPac 8200/8000 ML, Max Vision LLC, Madison, AL, USA) was used to measure CXCR7 staining intensity. The staining intensity criteria of positive cells was as follows: 0 , no staining; 1 , light yellow staining; 2 , brown staining; and 3 , dark brown staining. The scoring was performed by two independent pathologists based on the aforementioned color category. The percentages of positively stained cells were also considered in the evaluations. Thus, a score of 0 was assigned to samples with no positive cells; samples with $<10 \%$ of positive cells received a score of 1 ; those with $10-50 \%$ of positive cells received a score of 2 ; those with $51-80 \%$ of positive cells received a score of 3 ; and those with $>80 \%$ of positive cells received a score of 4 . Finally, these two values (staining intensity and percentage of positive cells) were multiplied to classify the specimens into CXCR7 low (final score, 0-6) or high (final score, 7-12) expression. Hematoxylin and eosin (H\&E) staining was performed to monitor tissue morphology.

Cell culture and transfection. The melanoma cell lines A375 and M14 were purchased from the American Type Culture Collection (Manassas, VA, USA) (16,17). The cells were grown in Dulbecco's modified Eagle's medium (DMEM) containing $10 \%$ fetal bovine serum (FBS) (both from Gibco; Thermo Fisher Scientific, Inc., Waltham, MA, USA) in an incubator at $37^{\circ} \mathrm{C}$ and $5 \% \mathrm{CO}_{2}$.

For transfection, cells were seeded in 24-well plates. To assess the effects of CXCR7 on the biological function of M14 cells (M14 was selected for its higher level of CXCR7 compared with A375 cell), CXCR7 small hairpin RNA (shRNA) or empty vectors (Genscript Biotech Corporation, Nanjing, China) were transfected into M14 cells using FuGENE HD Transfection Reagent (Promega Corporation, Madison, WI, USA). M14 cells were selected for their higher level of CXCR7 expression compared with A375 cells. The antibiotic G418 (1,000 $\mu \mathrm{g} / \mathrm{ml})$ (Invitrogen; Thermo Fisher Scientific, Inc.) was used for 2 weeks for cell selection upon shRNA transfection. CXCR7 shRNA- or vector shRNA-transfected M14 cells were subcloned and selected under a light microscope (IX51; Olympus Corporation, Tokyo, Japan). The selected CXCR7 shRNA- and vector shRNA-transfected M14 cell clones were cultured in $500 \mu \mathrm{g} / \mathrm{ml} \mathrm{G} 418$ for further experiments.

CXCR7 shRNA design and reverse transcription-quantitative polymerase chain reaction $(R T-q P C R)$. CXCR7 short interfering (si)RNA sequence was obtained from Thermo Fisher Scientific, Inc. (Silencer ${ }^{\circledR}$ Select siRNAs). CXCR7 shRNA was constructed based on the siRNA sequence by Genscript Biotech Corporation (Nanjing, China). Three pairs of shRNA were designed, and the pair with the best shRNA efficiency was selected. Western blotting was performed to confirm CXCR7 protein knocked down. Total RNA from cells was isolated using TRIzol reagent (Invitrogen; Thermo Fisher Scientific, Inc.). Total RNA quality and concentration were measured by ultraviolet spectrophotometry. The absorbance (A) 260/A280 range was set at 1.9-2.1 to assure good RNA quality. RNA (1 $\mu \mathrm{g} / \mathrm{sample}$ ) was used for RT to synthesize complementary DNA using random primers provided in the High-Capacity cDNA Reverse Transcription kit (Applied Biosystems; Thermo Fisher Scientific, Inc.). qPCR was performed to determine CXCR7 and $\beta$-actin messenger RNA (mRNA) expression. CXCR7 primers [CXCR-forward (F), 5'-CCCTGCATCCAT TCTCTCTT-3' and CXCR7-reverse (R), 5'-CCTGTTGCA AAACTGTCAGC-3'] and $\beta$-actin primers ( $\beta$-actin-F, 5'-GAA 
GGATTCCTATGTGGGCGAC-3' and $\beta$-actin-R, 5'-AGC CTGGATAGCAACGTACATGG-3') were used for qPCR using a PCR kit (GoTaq ${ }^{\circledR}$ Real-Time qPCR and RT-qPCR Systems for Dye-Based Detection, Promega Corporation). The reactions were performed under the following conditions: $95^{\circ} \mathrm{C}$ for $3 \mathrm{~min}$, followed by 45 cycles at $94^{\circ} \mathrm{C}$ for $20 \mathrm{sec}, 60^{\circ} \mathrm{C}$ for $30 \mathrm{sec}$ and $72^{\circ} \mathrm{C}$ for $20 \mathrm{sec}$. CXCR7 mRNA levels obtained from $\mathrm{qPCR}$ were normalized to $\beta$-actin mRNA levels yielding arbitrary units (18).

Western blotting. Upon harvesting, M14 or A375 cells were washed with PBS, and whole cell protein samples were extracted with radioimmunoprecipitation assay buffer [0.5\% Nonidet P-40, $10 \mathrm{mM}$ Tris ( $\mathrm{pH} 7.4), 150 \mathrm{mM} \mathrm{NaCl}$ and $1 \%$ SDS] containing a Protease Inhibitor Cocktail (Sigma-Aldrich; Merck KGaA, Darmstadt, Germany). Proteins $(70 \mu \mathrm{g} /$ well) were separated by $10 \%$ SDS-PAGE and transferred to polyvinylidene difluoride membranes. The primary antibodies used for western blotting were CXCR7 antibody () (cat. no., PA3-069, Thermo Fisher Scientific, Inc., dilution, 1:2,000) and mouse anti $\beta$-actin antibody (cat. no., sc-8432; Santa Cruz, Biotechnology, Inc., Dallas, TX, USA; dilution, 1:5,000). Each membrane was incubated with one primary antibody (in 5\% BSA) at $4^{\circ} \mathrm{C}$ in a cold room overnight. The secondary antibody ECL horseradish peroxidase-conjugated sheep anti-mouse IgG (cat. no., NA931; dilution, 1:5,000), or ECL HRP-conjugated donkey anti-rabbit IgG (cat. no., NA934V; dilution, 1:5,000) (GE Healthcare Life Sciences, Chalfont, UK) was incubated with the membrane at room temperature $\left(25^{\circ} \mathrm{C}\right)$ for $1 \mathrm{~h}$. Amersham ECL Prime Western Blotting Detection Reagent (GE Healthcare Life Sciences) was used to detect the chemiluminescent signals in an X-ray film.

Transwell chamber experimental settings. The Transwell chamber was purchased from Corning Incorporated (Corning, NY, USA), and included a polycarbonate membrane coated with matrigel that acted as an artificial basement membrane as previous described (15). The 24-well Transwell chamber was first incubated with $500 \mu 1$ DMEM without FBS for $2 \mathrm{~h}$. The medium was then replaced with $500 \mu \mathrm{l}$ cells in $10 \%$ FBS-DMEM $\left(0.6 \times 10^{6}\right.$ cells $\left./ \mathrm{ml}\right)$. These cells included non-transfected, empty vector shRNA-transfected and CXCR7 shRNA-transfected M14 cells (M14 cell was selected for its higher CXCR7 level compared to A375 cells) (300,000 cells/well), which were seeded in the upper chamber. Recombinant Human CXCR12 (cat. no., 350-NS/CF, R\&D Systems Inc., Minneapolis, MN, USA) (10 ng/ml) was added to all wells. The cells were incubated in the Transwell chamber for $24 \mathrm{~h}$. The bottom chamber of the Transwell membrane was then stained with Cell Counting kit-8 (Dojindo Molecular Technologies, Inc., Kumamoto, Japan) for $20 \mathrm{~min}$. The cells were next washed with PBS to remove the excess dye and air dried for $1 \mathrm{~h}$. The number of cells that passed through the membrane was then counted under a light microscope (IX51; Olympus Corporation, Tokyo, Japan). All experiments were repeated three times.

Animal studies. All animal experiments were approved by the Chinese Academy of Medical Sciences and Affiliated Hospital of Hebei University Animal Policy and Welfare Committee, and complied with the National Institutes of Health guidelines (Guide for the Care and Use of Laboratory Animals) (https://www.ncbi.nlm.nih.gov/books/NBK54050/). Wild type BALB/c nude mice were obtained from Shanghai Laboratory Animal Center (Shanghai, China). There were 18 mice ( 5 weeks old; 9 female, 9 male; average weight $18-19 \mathrm{~g}$ ) used in the experiment. The mice were housed at temperatures $\sim 23^{\circ} \mathrm{C}$ with $40-60 \%$ humidity and $12 \mathrm{~h}$ light $/ 12 \mathrm{~h}$ dark cycle. Food and water were accessible at libitum. The effects of CXCR7 on the tumor progression of skin melanoma were tested in transplanted mice. Untreated, empty vector shRNA-transfected and CXCR7 shRNA-transfected M14 cells were harvested. Each cell suspension $\left(1 \times 10^{6}\right.$ cells $\left./ 100 \mu \mathrm{l}\right)$ was subcutaneously inoculated into the mice right armpit. Tumor size was measured from day 11 following tumor transplantation. Tumor volume (TV) was calculated by the following formula: $T V=1 / 2 x a b^{2}$, where $\mathrm{a}$ and $\mathrm{b}$ are the tumor length and width, respectively. Mice were sacrificed by carbon dioxide at day 21 post-tumor transplantation. The 201 plastid chamber with a lid was used for mice euthanasia. Compressed $\mathrm{CO}_{2}$ gas in cylinders was used for source of $\mathrm{CO}_{2}$ with gas inflow to the chamber regulated at a speed $\sim 30-50 \%$ of the chamber or cage volume/min to provide a final concentration of $50 \%$ for $\sim 1 \mathrm{~min}$. After the gas was stopped, the animal was then observed until all muscle activity and signs of life have been absent for $\geq 30 \mathrm{sec}$. The tumors were harvested, weighted and recorded. The maximum weight was $\sim 1.70 \mathrm{~g}$.

Statistical analysis. SPSS statistical software (version 13.0; SPSS, Inc., Chicago, IL, USA) was used for data analysis. Data are expressed as the mean \pm standard deviation of $\geq 3$ sample replicates, unless stated otherwise. ${ }^{*} \mathrm{P}<0.05,{ }^{* *} \mathrm{P}<0.01$, or ${ }^{* * *} \mathrm{P}<0.001$ was considered to indicate a statistically significant difference.

\section{Results}

CXCR7 expression in three types of skin cancer tissue. Immunohistochemistry staining was performed to assess the expression of CXCR7 in skin cancer tissues. H\&E staining was also performed to monitor cancer tissue morphology (Fig. 1A-C). CXCR7 protein-positive samples were stained in brown color. CXCR7 protein was located in the cytoplasm and cell membrane (Fig. 1A-C). CXCR7 protein expression levels were compared between SCC, BCC and MM, i.e., the three types of skin cancer tissues evaluated in the present study (Table I). It was observed that MM had the highest (80\%, 24 out of 30 cases) percentage of CXCR7 protein expression, followed by SCC-L with $38.9 \%$. SCC-H and BCC had 8.3 and $8.0 \%$ of high CXCR7 protein expression levels, respectively (Tables I and II). These findings indicated that CXCR7 is involved in tumor progression.

CXCR7 expression in melanoma cell lines M14 and A375. Western blotting was performed to determine the CXCR7 protein expression levels in melanoma M14 and A375 cells. $\beta$-actin protein expression was used as a control. The CXCR7/ $\beta$-actin protein ratio indicated that M14 cells had much higher CXCR7 protein levels compared with those of 
Table I. Patient's CXCR7 protein levels in SCC, MCC and MM cases, according to their pathology details and anti-CXCR7 staining score.

\begin{tabular}{|c|c|}
\hline Pathology number & Anti-CXC \\
\hline \multicolumn{2}{|l|}{$\mathrm{SCC}$} \\
\hline \multicolumn{2}{|l|}{14792} \\
\hline \multicolumn{2}{|l|}{14615} \\
\hline \multicolumn{2}{|l|}{14552} \\
\hline \multicolumn{2}{|l|}{14502} \\
\hline \multicolumn{2}{|l|}{14365} \\
\hline \multicolumn{2}{|l|}{14325} \\
\hline \multicolumn{2}{|l|}{14298} \\
\hline \multicolumn{2}{|l|}{14147} \\
\hline \multicolumn{2}{|l|}{14143} \\
\hline \multicolumn{2}{|l|}{14005} \\
\hline \multicolumn{2}{|l|}{13945} \\
\hline \multicolumn{2}{|l|}{13911} \\
\hline \multicolumn{2}{|l|}{13905} \\
\hline \multicolumn{2}{|l|}{13874} \\
\hline \multicolumn{2}{|l|}{13866} \\
\hline \multicolumn{2}{|l|}{13759} \\
\hline \multicolumn{2}{|l|}{12114} \\
\hline \multicolumn{2}{|l|}{12203} \\
\hline \multicolumn{2}{|l|}{12102} \\
\hline \multicolumn{2}{|l|}{12009} \\
\hline \multicolumn{2}{|l|}{11658} \\
\hline \multicolumn{2}{|l|}{11411} \\
\hline \multicolumn{2}{|l|}{11402} \\
\hline \multicolumn{2}{|l|}{11252} \\
\hline \multicolumn{2}{|l|}{10892} \\
\hline \multicolumn{2}{|l|}{10598} \\
\hline \multicolumn{2}{|l|}{10454} \\
\hline \multicolumn{2}{|l|}{10336} \\
\hline \multicolumn{2}{|l|}{10215} \\
\hline \multicolumn{2}{|l|}{10112} \\
\hline $\mathrm{BCC}$ & \\
\hline
\end{tabular}

14955

14912

14905

14478

14117

13819

13856

13848

13722

13692

13189

12751

12502

12452

12441

12438

12098

11791
Table I. Continued.

Pathology number

Anti-CXCR7 score

$\begin{array}{ll}11302 & 4 \\ 11217 & 4 \\ 11009 & 4 \\ 10953 & 8 \\ 10255 & 0 \\ 10233 & 1 \\ 10115 & 3\end{array}$

MM

$14576 \quad 8$

$14291 \quad 8$

$14002 \quad 8$

$13879 \quad 3$

$13762 \quad 8$

$13412 \quad 9$

$13255 \quad 9$

$12908+9$

$12745+9$

$12702 \quad 8$

$12537 \quad 6$

$12524 \quad 8$

$12515 \quad 8$

$12355 \quad 8$

$12118 \quad 8$

$12035 \quad 8$

$11387 \quad 8$

$10652 \quad 9$

$10557 \quad 4$

$10102 \quad 9$

$09711 \quad 9$

$09658+9$

$09356 \quad 2$

$09110 \quad 8$

$09005 \quad 9$

$08562 \quad 4$

$08312+4$

$08254 \quad 8$

CXCR7, C-X-C chemokine receptor type 7; SCC, squamous cell carcinoma; BCC, basal cell carcinoma; MM, malignant melanoma.

A375 cells (Fig. 2A and B). Therefore, M14 cells were selected for our experiments.

CXCR7 shRNA significantly reduces CXCR7 $m R N A$ and protein expression in $M 14$ cells. shRNA was used to knock down CXCR7 expression in M14 cells. G418 was used for clone selection following shRNA transfection. Green fluorescent protein observation by fluorescence microscopy indicated that $95 \%$ of cells were transfected with the CXCR7 shRNA or empty vector. qPCR data revealed that CXCR7 shRNA significantly inhibited CXCR7 mRNA expression in M14 cells 
A
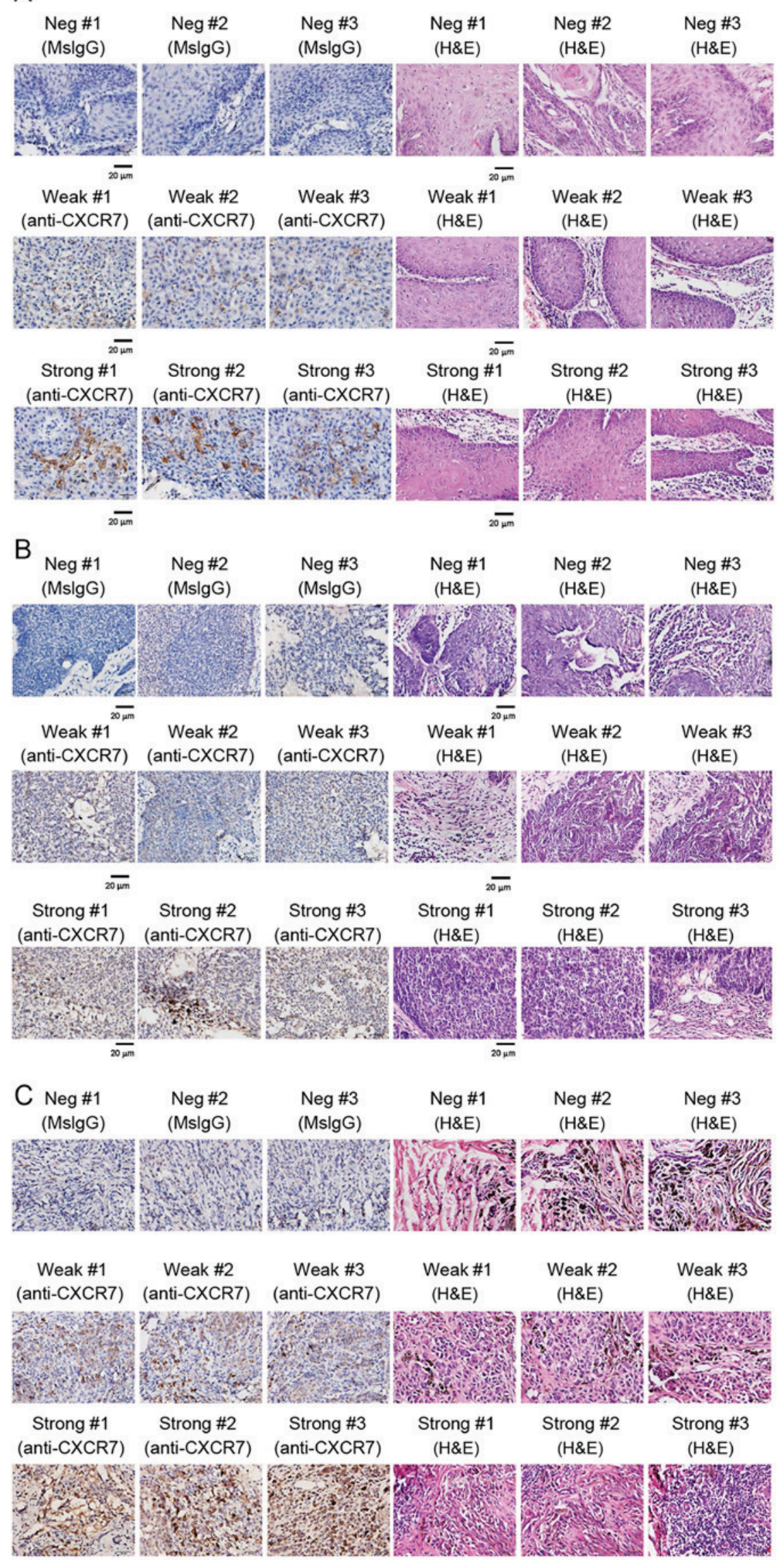

Figure 1. Immunohistochemistry staining of CXCR7 protein in skin cancer tissues. Three selected images of negative (MsIgG) control, weak and strong CXCR7 staining, and their H\&E staining were presented for each cancer category. Representative images of staining with MsIgG, anti-CXCR7 or H\&E for (A) squamous cell carcinoma, (B) basal cell carcinoma and (C) malignant melanoma tissues. Positive CXCR7 staining appears as a brown color in the cytoplasm. Scale bar, $20 \mu \mathrm{m}$. MsIgG, mouse immunoglobulin G; CXCR7, C-X-C chemokine receptor type 7; H\&E, hematoxylin and eosin. 
A

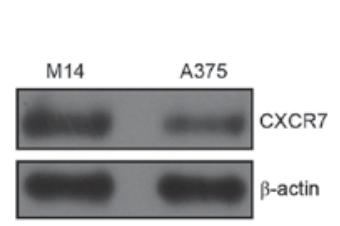

B

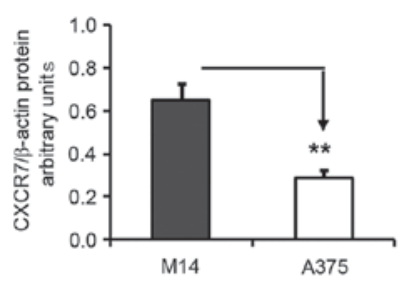

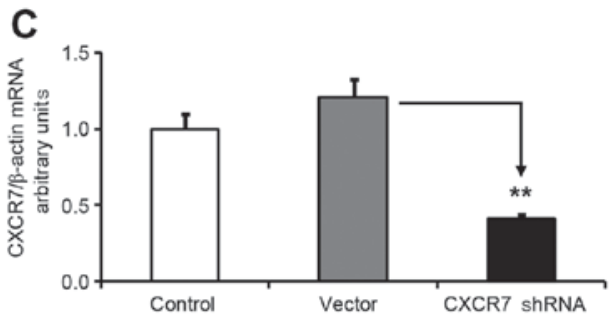

D

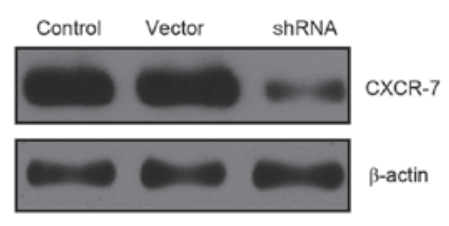

E

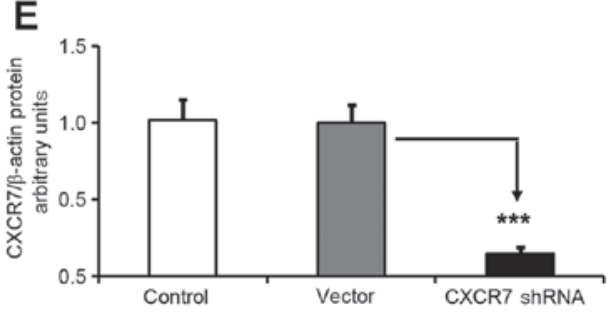

Figure 2. CXCR7 shRNA knocks down CXCR7 mRNA and protein expression levels in melanoma M14 cells. (A) Western blot analysis of CXCR7 protein expression levels in melanoma M14 and A375 cells. $\beta$-actin was used as a protein level control. (B) M14 cells had significantly higher CXCR7 protein expression compared with that of A375 cells. The CXCR7/ $\beta$-actin protein ratio in M14 and A375 cells was $0.649 \pm 0.080$ and $0.288 \pm 0.040$, respectively $\left({ }^{* *} \mathrm{P}=0.006\right)$. (C) CXCR7 shRNA significantly reduced CXCR7 mRNA expression in M14 cells. CXCR7 shRNA knock down was performed in M14 cells. Empty vector-transfected and non-transfected M14 cells were used as controls. Polymerase chain reaction was conducted to monitor CXCR7 mRNA expression. $\beta$-actin was used as an internal mRNA level control. The CXCR7/ $\beta$-actin mRNA ratio was $1.000 \pm 0.102$ (control), $1.211 \pm 0.117$ (vector-transfected) and $0.412 \pm 0.023$ (CXCR7 shRNA-transfected) in M14 cells. ( ${ }^{* *} \mathrm{P}=0.007$ was obtained for the comparison between vector-transfected and CXCR7 shRNA-transfected cells). (D) Western blot images of CXCR7 and $\beta$-actin protein expression levels. (E) CXCR7 shRNA significantly inhibited CXCR7 protein expression in M14 cells. The CXCR7/ $\beta$-actin protein ratio was $1.000 \pm 0.002$ (control), $1.016 \pm 0.004$ (vector) and $0.144 \pm 0.005$ (CXCR7 shRNA) in the different groups, which confirmed the knockdown of CXCR7 protein expression in M14 cells $\left(^{* * *} \mathrm{P}<0.001\right.$ was obtained for the comparison between vector and CXCR7 shRNA groups). CXCR7, C-X-C chemokine receptor type 7; shRNA, small hairpin RNA; mRNA, messenger RNA.

Table II. Summary of CXCR7 protein levels in SCC, MCC and MM patients.

\begin{tabular}{lcccc}
\hline & & \multicolumn{3}{c}{ CXCR7 protein expression levels } \\
\cline { 3 - 5 } Group & Cases, $\mathrm{n}$ & Low, $\mathrm{n}$ & High, $\mathrm{n}$ & High, \% \\
\hline SCC-T & 30 & 22 & 8 & 26.7 \\
SCC-H & 12 & 11 & 1 & 8.3 \\
SCC-L & 18 & 11 & 7 & 38.9 \\
BCC & 25 & 23 & 2 & 8.0 \\
MM & 30 & 6 & 24 & 80.0 \\
\hline
\end{tabular}

${ }^{\mathrm{a} C X C R 7}$ was classified as low expression (weak staining; score, 0-6) or high expression (strong staining; score, 7-12). The percentage of high CXCR7 expression cases was calculated as the number of high CXCR7 expression cases divided by the total number of cases and multiplied by 100 . CXCR7, C-X-C chemokine receptor type 7; SCC, squamous cell carcinoma; SCC-T, total SCC; SCC-H, high differentiated SCC; SCC-L, low differentiated SCC; BCC, basal cell carcinoma; MM, malignant melanoma.

(Fig. 2C). Western blotting for CXCR7 and $\beta$-actin further confirmed the knocked down of CXCR7 protein expression in M14 cells (Fig. 2D and E).

CXCL12 cytokine induces M14 cell invasion. Cells that are able to pass through a polycarbonate membrane in matrigel Transwell chamber assays are used to study cell migration and invasion (15). CXCL12 has been demonstrated to stimulate cell biological functions $(10,19,20)$. In the present study,
CXCL12 effects on M14 cell invasive activity were tested in a matrigel Transwell chamber assay. The M14 cells that passed through the Transwell chamber and bound to the matrigel were counted. It was observed that both 10 and $100 \mathrm{ng} / \mathrm{ml} \mathrm{CXCL12}$ significantly increased the number of M14 cells that passed through the Transwell chamber, thus confirming that CXCL12 induced M14 cell invasive ability (Fig. 3A and B).

CXCR7 knockdown reduces CXCL12-induced invasive activity in M14 cells. The present study used $10 \mathrm{ng} / \mathrm{ml} \mathrm{CXCL12} \mathrm{to}$ assess the CXCR7 biological function in M14 cells. The M14 cell invasive ability was monitored in a matrigel Transwell chamber assay with CXCR7 shRNA-transfected, empty vector shRNA-transfected and non-transfected M14 cells. It was observed that CXCR7 knockdown significantly reduced the invasive activity of M14 cells compared with that of shRNA vector-transfected and control cells. By contrast, there was not a significant difference in the number of M14 cells between shRNA vector-transfected and control cells (Fig. 3C and D). These data indicated that CXCR7 may regulate M14 cell migration and invasion.

CXCR7 shRNA inhibits melanoma tumor development in mice. The effects of CXCR7 on transplanted skin melanoma tumor progression were tested in mice. The transplanted tumors were monitored daily, and the sizes were measured from day 11 to day 21 post-transplantation. It was observed that the tumor size grew continually in the control and vector groups. By contrast, the tumor size in the mice transfected with CXCR7 shRNA did not grow as much as that of the control or shRNA vector-transfected mice (Fig. 4). Tumors were harvested at day 21 post-implantation. Smaller tumor sizes were observed 
A

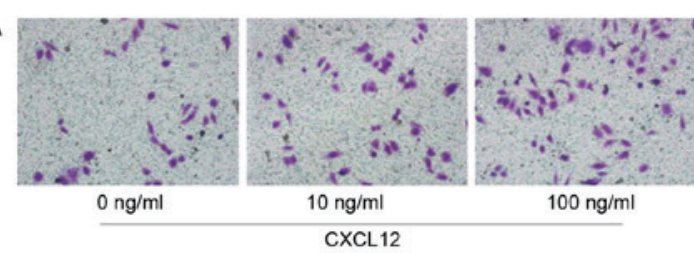

B

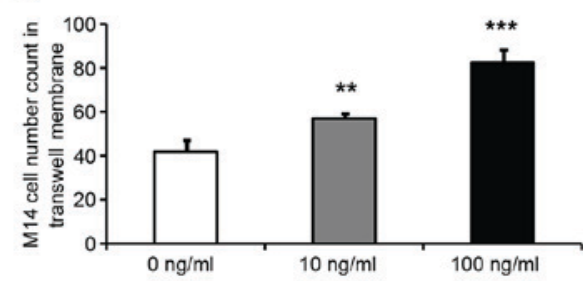

C

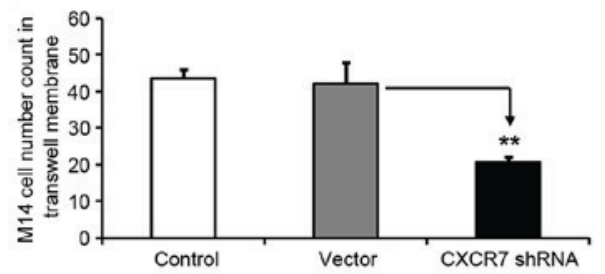

D

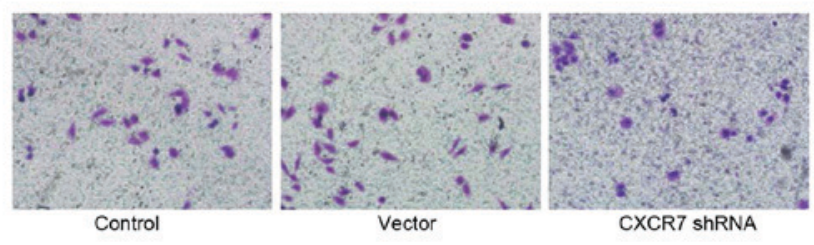

Figure 3. CXCL12 cytokine induces M14 cell invasive activity, and CXCR7 knocked down inhibits CXCR12-induced M14 cell invasive activity. M14 cells $(10,000$ cells $/ 2 \mathrm{ml})$ were seeded in each Transwell chamber with 0,10 or $100 \mathrm{ng} / \mathrm{ml}$ CXCL12 for 24 h. The cells that passed through the Transwell chamber and bound to the matrigel were recorded and counted. (A) Images of M14 cells bound to the matrigel membrane (magnification, $\mathrm{x} 400$ (B) CXCL12 increased M14 cell invasive activity. The M14 cell count in the Transwell matrigel membrane was 41.67 \pm 5.51 (0 ng/ml CXCL12), 56.67 $\pm 2.52(10 \mathrm{ng} / \mathrm{ml} \mathrm{CXCL12)} \mathrm{and}$ $82.23 \pm 6.11$ (100 ng/ml CXCL12). P-values were obtained for the comparisons between the indicated treatments and $0 \mathrm{ng} / \mathrm{ml} \mathrm{CXCR} 12\left({ }^{* *} \mathrm{P}<0.01\right.$; $\left.{ }^{* * *} \mathrm{P}<0.001\right)$. (C) CXCR7 shRNA significantly reduced M14 cell counts in in the matrigel membrane. The M14 cell count in the Transwell matrigel membrane was $43.63 \pm 2.15$ (control), $42.00 \pm 6.02$ (vector) and 20.62 \pm 1.50 (CXCR7 shRNA). ${ }^{* *} \mathrm{P}<0.01$ was obtained for the comparison between vector and CXCR7 shRNA. There were not significantly different cell counts between the control and vector groups. These results indicated that CXCR7 downregulation inhibited M14 cell migration and invasion. (D) Images of M14 cells in the matrigel membrane (magnification, x400). Untreated M14 cells (control), empty vector shRNA-transfected M14 cells (vector) and CXCR7 shRNA-transfected M14 cells (10,000 cells/2 ml/well) were plated in Transwell chamber wells with 10 ng/ml CXCR12 for 24 h. The cells that passed through the membrane and bound to the matrigel were recorded and counted. A lower cell number was observed in CXCR7 shRNA-transfected M14 cells compared with that in control and vector-transfected M14 cells. CXCR7, C-X-C chemokine receptor type 7; shRNA, small hairpin RNA; CXCL12, C-X-C motif chemokine ligand 12.

in CXCR7 shRNA mice compared with those in control and shRNA vector mice (Fig. 5A and B). It has been reported that vasculature serves an important role in skin cancer inflammation (21), therefore the tumor weight and tumor vascular number were also compared. The average tumor weight was significantly lower in the CXCR7 shRNA group compared with that in the control and vector shRNA groups $\left({ }^{*} \mathrm{P}<0.05\right.$; Fig. 5C). In addition, the tumor vascular number was also significantly lower in CXCR7 shRNA mice compared with that in vector shRNA and control mice $\left({ }^{* *} \mathrm{P}<0.01\right.$; Fig. $\left.5 \mathrm{D}\right)$. These findings further demonstrated the effects of CXCR7 on tumor development in a mouse model.

\section{Discussion}

In the present study, a higher percentage $(80 \%)$ of positive CXCR7 protein staining was observed in MM tumors compared with that in SSC and BCC tumors from patients, indicating CXCR7 involvement in melanoma cancer progression and development. It was observed that M14 melanoma cells expressed high levels of CXCR7. CXCR7 shRNA significantly knocked down CXCR7 mRNA and protein expression in M14 cells, which subsequently inhibited M14 cell biological functions, including cell migration, invasion and metastasis. There are several reports indicating that CXCR7 expression was significantly higher in tumor tissue than in adjacent normal tissue $(10,22)$. The higher rate of cancer spreading to other parts of the body was associated with higher CXCR7 levels in cancer (23), further supporting the CXCR7-mediated induction of skin melanoma invasion and metastasis.

Several studies have shown that melanoma cell lines co-express high levels of chemokine factor receptors and

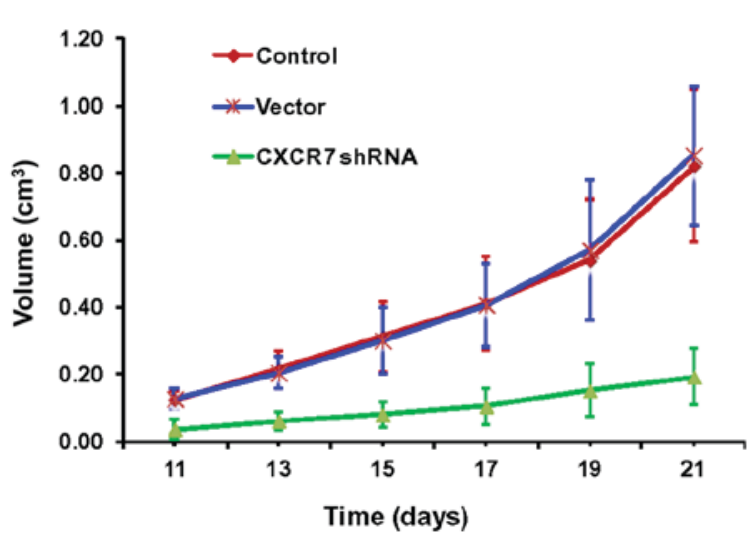

Figure 4. Progression of transplanted tumor volume with time in mice. Comparisons between control, vector and CXCR7 shRNA treatments. The tumor sizes increased daily over time following transplantation in the control and vector mice. However, the tumor volumes were smaller in CXCR7 shRNA mice compared with those in the control and vector mice from day 11 to day 21 post-implantation. There was not difference in tumor size between the control and vector groups. CXCR7, C-X-C chemokine receptor type 7; shRNA, small hairpin RNA.

ligands (24). Chemokines and tumor cell surface receptors are involved in post-melanoma angiogenesis, tumor cell growth, invasion and metastasis of malignant processes (25). Furthermore, high levels of cell surface CXCR4 expression were associated with melanoma tumor surface ulceration, tumor invasion and increased mortality (26). CXCR3 expression levels were associated with tumor cell infiltration depth in primary invasive melanoma (27). High expression of CXCR3 and its ligands CXCL9 and CXCL10 led to actin polymerization 
A
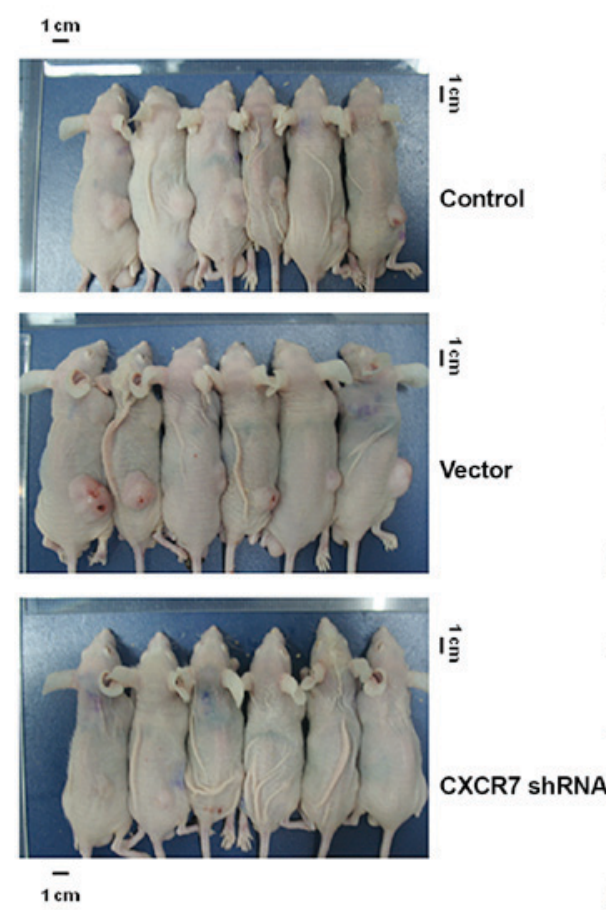

B

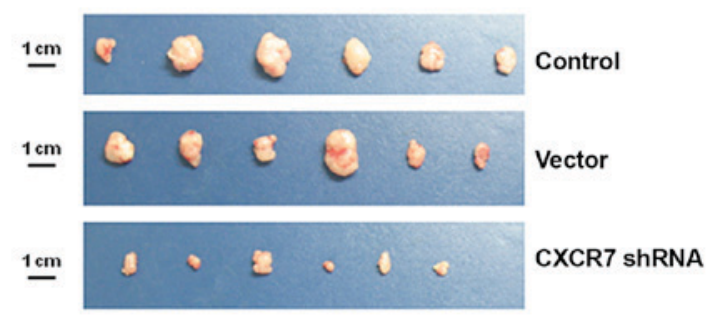

C

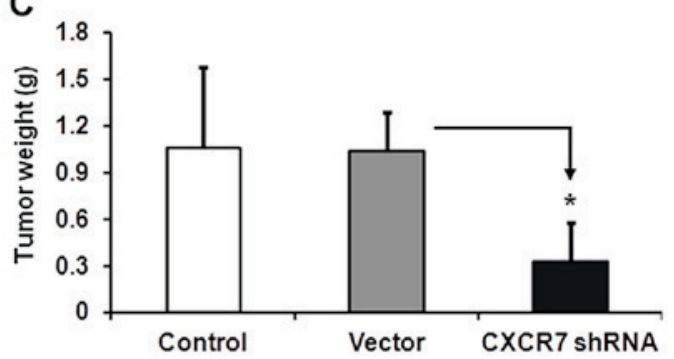

D

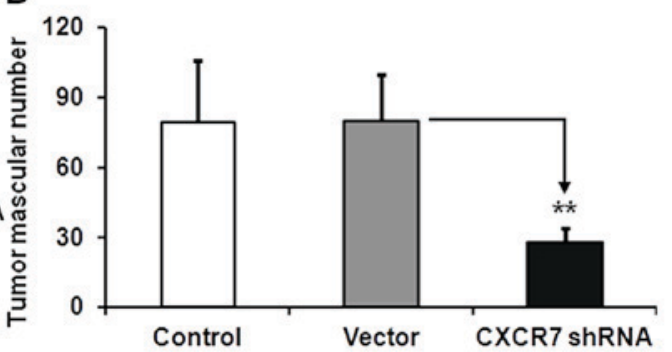

Figure 5. CXCR7 shRNA significantly inhibits transplanted tumors in mice. (A) Transplanted tumor morphology inside the mice skin. The tumor sizes were visually larger in the control and vector-transfected mice compared with those in the CXCR7 shRNA-transfected mice at day 21 post-implantation. Scale bar, $1 \mathrm{~cm}$. (B) Images if harvested tumors from control, vector and CXCR7 shRNA mice. Markedly smaller tumor sizes were observed in CXCR7 shRNA-transfected mice compared with those in control and vector-transfected mice. Scale bar, $1 \mathrm{~cm}$. (C) CXCR7 shRNA significantly reduced tumor weight. Tumor weight was significantly smaller in CXCR7 shRNA mice compared with that in control and vector mice. The tumor weight was $1.06 \pm 0.52 \mathrm{~g}$ (control), $1.04 \pm 0.25 \mathrm{~g}$ (vector) and $0.33 \pm 0.24 \mathrm{~g}$ (CXCR7 shRNA). ${ }^{*} \mathrm{P}<0.05$ was obtained for the comparison between vector and CXCR7 shRNA groups. (D) CXCR7 shRNA significantly inhibited the tumor vascular number. The number of vascular tumors in the transplanted mice was counted. A significantly lower number of vascular tumors was observed in CXCR7 shRNA mice compared with that in control and vector mice. The tumor vascular number was $79 \pm 27$ (control), $80 \pm 20$ (vector) and $28 \pm 6$ (CXCR7 shRNA). ${ }^{* *} \mathrm{P}<0.01$ was obtained for the comparison between vector and CXCR7 shRNA groups. CXCR7, C-X-C chemokine receptor type 7; shRNA, small hairpin RNA.

and invasion in B16F10 murine melanoma models $(26,27)$. The expression of the chemokine receptors CCR7, CCR10, CXCR3 and CXCR4 was associated with melanoma lymph node metastasis in mouse models $(19,23,27,28)$. CXCR7 interaction with the CXCL12 receptor has been demonstrated to increase the CXCL12-induced normal epidermal cell migration (29). Furthermore, it was observed that the chemokine ligand for CXCR7 was CXCL12 (also known as SDF-1), which also binds CXCR4 (30). CXCR7 has been demonstrated to heterodimerize with CXCR4 and regulate CXCL12-mediated $\mathrm{G}$ protein signaling (28). These findings supported CXCR7 roles on melanoma biological behavior. CXCR7 is involved in multiple functions, including tumor growth, survival and metastasis, in several human tumors such as breast cancer, pancreatic cancer, papillary thyroid carcinoma and prostate cancer $(19,20,31)$. High CXCR7 expression may promote angiogenesis, enhance tumor cell invasion, and promote tumor invasion and metastasis $(10,22,28,29)$. Hypoxia-inducible factor- $1 \alpha$ has been demonstrated to increase CXCR7 expression and activate epidermal growth factor receptor signal transduction, and subsequently promote the growth of cancer cells in prostate cancer (32). It has been demonstrated that overexpression of
CXCR7 in malignant human myeloid cells resulted in nuclear factor- $\kappa \mathrm{B}$ activation, followed by mitogen-activated protein kinase p42/44 and AKT phosphorylation, and subsequently increased cancer cell migration (22).

Therefore, a model can be proposed in which CXCR7 interacts with CXCL12 to activate the chemokine receptor signaling pathway, and to increase melanoma cell migration and invasion. It can be speculated that CXCR7 is a potential therapeutic target for cutaneous melanoma treatments. Further characterization of CXCR7-induced chemokine receptor signaling pathways is warranted.

\section{References}

1. Balch CM, Soong SJ, Gershenwald JE, Thompson JF, Reintgen DS, Cascinelli N, Urist M, McMasters KM, Ross MI, Kirkwood JM, et al: Prognostic factors analysis of 17,600 melanoma patients: Validation of the American Joint Committee on Cancer melanoma staging system. J Clin Oncol 19: 3622-3634, 2001.

2. Balch CM, Soong SJ, Smith T, Ross MI, Urist MM,Karakousis CP, Temple WJ, Mihm MC, Barnhill RL, Jewell WR, et al: Long-term results of a prospective surgical trial comparing $2 \mathrm{~cm}$ vs. $4 \mathrm{~cm}$ excision margins for 740 patients with $1-4 \mathrm{~mm}$ melanomas. Ann Surg Oncol 8: 101-108, 2001. 
3. Slominski A, Wortsman J, Carlson AJ, Matsuoka LY, Balch CM and Mihm MC: Malignant melanoma. Arch Pathol Lab Med 125: 1295-1306, 2001.

4. Balch CM, Buzaid AC, Soong SJ, Atkins MB, Cascinelli N, Coit DG, Fleming ID, Gershenwald JE, Houghton A Jr, Kirkwood JM, et al: Final version of the American Joint Committee on Cancer staging system for cutaneous melanoma. J Clin Oncol 19: 3635-3648, 2001.

5. DeSantis CE, Lin CC, Mariotto AB, Siegel RL, Stein KD, Kramer JL, Alteri R, Robbins AS and Jemal A: Cancer treatment and survivorship statistics, 2014. CA Cancer J Clin 64: 252-271, 2014.

6. Morton DL, Wen DR, Wong JH, Economou JS, Cagle LA, Storm FK, Foshag LJ and Cochran AJ: Technical details of intraoperative lymphatic mapping for early stage melanoma. Arch Surg 127: 392-399, 1992.

7. Baggiolini M and Loetscher P: Chemokines in inflammation and immunity. Immunol Today 21: 418-420, 2000.

8. Balkwill F: Cancer and the chemokine network. Nat Rey Cancer 4: 540-550, 2004

9. Rossi D and Zlotnik A: The biology of chemokines and their receptors. Annu Rev Immunol 18: 217-242, 2000.

10. Sánchez-Martin L, Sánchez-Mateos $P$ and Cabañas C: CXCR7 impact on CXCL12 biology and disease. Trends Mol Med 19: $12-22,2013$.

11. Beutner KR, Geisse JK, Helman D, Fox TL, Ginkel A and Owens ML: Therapeutic response of basal cell carcinoma to the immune response modifier imiquimod 5\% cream. J Am Acad Dermatol 41: 1002-1007, 1999

12. Rowe DE, Carroll RJ and Day CL Jr: Prognostic factors for local recurrence, metastasis, and survival rates in squamous cell carcinoma of the skin, ear, and lip. Implications for treatment modality selection. J Am Acad Dermatol 26: 976-990, 1992.

13. Shaw LM: Tumor cell invasion assays. Methods Mol Biol 294: 97-105, 2005

14. Yang FC, Atkinson SJ, Gu Y, Borneo JB, Roberts AW, Zheng Y, Pennington J and Williams DA: Rac and Cdc42 GTPases control hematopoietic stem cell shape, adhesion, migration, and mobilization. Proc Natl Acad Sci USA 98: 5614-5618, 2001.

15. Tsareva SA, Moriggl R, Corvinus FM, Wiederanders B Schütz A, Kovacic B and Friedrich K: Signal transducer and activator of transcription 3 activation promotes invasive growth of colon carcinomas through matrix metalloproteinase induction. Neoplasia 9: 279-291, 2007

16. Giard DJ, Aaronson SA, Todaro GJ, Arnstein P, Kersey JH, Dosik $\mathrm{H}$ and Parks WP: In vitro cultivation of human tumors: Establishment of cell lines derived from a series of solid tumors. J Natl Cancer Inst 51: 1417-1423, 1973.

17. Sulit HL, Golub SH, Irie RF, Gupta RK, Grooms GA and Morton DL: Human tumor cells grown in fetal calf serum and human serum: Influences on the tests for lymphocyte cytotoxicity, serum blocking and serum arming effects. Int J Cancer 17: 461-468, 1976.

18. Larionov A, Krause A and Miller W: A standard curve based method for relative real time PCR data processing. BMC Bioinformatics 6: 62, 2005.
19. Sun X, Cheng G, Hao M, Zheng J, Zhou X, Zhang J, Taichman RS Pienta KJ and Wang J: CXCL12/CXCR4/CXCR7 chemokine axis and cancer progression. Cancer Metastasis Rev 29: 709-722, 2010.

20. Wang J, Shiozawa Y, Wang J, Wang Y, Jung Y, Pienta KJ, Mehra R, Loberg R and Taichman RS: The role of CXCR7/RDC1 as a chemokine receptor for CXCL12/SDF-1 in prostate cancer. J Biol Chem 283: 4283-4294, 2008.

21. Huggenberger R and Detmar M: The cutaneous vascular system in chronic skin inflammation. J Investig Dermatol Symp Proc 15: 24-32, 2011.

22. Tarnowski M, Liu R, Wysoczynski M, Ratajczak J, Kucia M and Ratajczak MZ: CXCR7: A new SDF-1-binding receptor in contrast to normal CD34(+) progenitors is functional and is expressed at higher level in human malignant hematopoietic cells. Eur J Haematol 85: 472-483, 2010.

23. D'Alterio C, Consales C, Polimeno M, Franco R, Cindolo L, Portella L, Cioffi M, Calemma R, Marra L, Claudio L, et al: Concomitant CXCR4 and CXCR7 expression predicts poor prognosis in renal cancer. Curr Cancer Drug Targets 10: 772-781, 2010.

24. Pinto S, Martinez-Romero A, O'Connor JE, Gil-Benso R, San-Miguel T, Terrádez L, Monteagudo $\mathrm{C}$ and Callaghan RC: Intracellular coexpression of CXC- and CC-chemokine receptors and their ligands in human melanoma cell lines and dynamic variations after xenotransplantation. BMC Cancer 14: 118, 2014.

25. Richmond A, Yang J and Su Y: The good and the bad of chemokines/chemokine receptors in melanoma. Pigment Cell Melanoma Res 22: 175-186, 2009.

26. Longo-Imedio MI, Longo N, Treviño I, Lázaro $P$ and Sánchez-Mateos P: Clinical significance of CXCR3 and CXCR4 expression in primary melanoma. Int J Cancer 117: 861-865, 2005.

27. Monteagudo C, Martin JM, Jorda E and Llombart-Bosch A: CXCR 3 chemokine receptor immunoreactivity in primary cutaneous malignant melanoma: Correlation with clinicopathological prognostic factors. J Clin Pathol 60: 596-599, 2007.

28. Levoye A, Balabanian K, Baleux F, Bachelerie F and Lagane B: CXCR7 heterodimerizes with CXCR4 and regulates CXCL12-mediated G protein signaling. Blood 113: 6085-6093, 2009.

29. Lee E, Han J, Kim K, Choi H, Cho EG and Lee TR: CXCR7 mediates SDF1-induced melanocyte migration. Pigment Cell Melanoma Res 26: 58-66, 2013.

30. Sierro F, Biben C, Martinez-Muñoz L, Mellado M, Ransohoff RM, Li M, Woehl B, Leung H, Groom J, Batten M, et al: Disrupted cardiac development but normal hematopoiesis in mice deficient in the second CXCL12/SDF-1 receptor, CXCR7. Proc Natl Acad Sci USA 104: 14759-14764, 2007.

31. Salazar N, Muñoz D, Kallifatidis G, Singh RK, Jordà M and Lokeshwar BL: The chemokine receptor CXCR7 interacts with EGFR to promote breast cancer cell proliferation. Mol Cancer 13: 198,2014

32. Singh RK and Lokeshwar BL: The IL-8-regulated chemokine receptor CXCR7 stimulates EGFR signaling to promote prostate cancer growth. Cancer Res 71: 3268-3277, 2011. 